\title{
Tumor Thrombus Involving the Inferior Vena Cava in Renal Malignancy: Is There a Difference in Clinical Presentation and Outcome among Right and Left Side Tumors?
}

\author{
Devendar Katkoori, Manoharan Murugesan, Gaetano Ciancio, Mark S. Soloway \\ Department of Urology, Miller School of Medicine, University of Miami, Miami, Florida, USA
}

\begin{abstract}
Purpose: Renal cell carcinoma (RCC) has a propensity to propagate into the renal vein and inferior vena cava (IVC). Due to inherent differences in the venous anatomy of the right and left kidneys, tumor thrombus involvement of IVC may vary. The aim of this study is to compare clinical presentation and outcome of right vs. left RCC with IVC thrombus. Materials and Methods: Patients who underwent radical nephrectomy and IVC thrombectomy between 1997 and 2008 were identified. All relevant data were collected and analyzed.

Results: Eight-seven patients were included. Sixty patients $(69 \%)$ had a right sided tumor. Mean tumor size was $10.2( \pm 4)$ $\mathrm{cm}$ and was not significantly different on either side. Fifty-six percent of right sided tumors had level-III (intra-hepatic) or higher tumor thrombus, while $22 \%$ of left sided tumors had similar level thrombus extension ( $p<0.0001$ ). Nearly $50 \%$ of left sided tumors showed level-I thrombus compared to $10 \%$ of right side tumors. A comparison of age, estimated blood loss and transfusion rate was not significantly different. The recurrence free $(p=0.9)$ and disease specific survival $(p=$ 0.4 ) were not significantly different between the right and left side tumors with IVC thrombus.

Conclusion: A level-III IVC tumor thrombus is more frequently seen with a right side tumor. However, clinical and operative characteristics among the left and right sided tumors with IVC thrombus were not different. More significantly, recurrence rate and survival did not differ with the laterality of the tumor.
\end{abstract}

Key words: kidney neoplasms; nephrectomy; vena cava, inferior; thrombectomy

Int Braz J Urol. 2009; 35: 652-7

\section{INTRODUCTION}

Renal cell carcinoma (RCC) accounts for $3.5 \%$ of all adult malignant neoplasms $(1,2)$. Intra-luminal tumor growth into renal venous system is one of the unique features of RCC. The tumor thrombus can involve the inferior vena cava(IVC) in $4 \%$ to $10 \%$ of patients (3). Radical nephrectomy and IVC thrombectomy offers reasonable long term survival. Although clinico-pathological outcome of these tumors with IVC thrombus has been well studied, comparisons between right and left sided tumors have not been previously reported.

Typically, the right renal vein is short and without any branches whereas the left renal vein is longer and receives the lumbar, gonadal and adrenal veins before draining into the IVC. Due to these inherent differences in the renal venous anatomy, the incidence of IVC involvement and the level of thrombus may vary depending on the laterality of the tumor. Whether this difference can lead to a significant difference in outcome for patients with 
IVC involvement with the tumor on right versus left has not been assessed. We retrospectively reviewed our nephrectomy database to see if laterality of the tumor had any bearing on the clinical presentation and outcome following radical nephrectomy and IVC thrombectomy.

\section{MATERIALS AND METHODS}

We retrospectively analyzed patients who underwent radical nephrectomy and IVC thrombectomy from 1997 to 2008 by our single surgical team. After obtaining Institutional Review Board approval, all the relevant data were collected and analyzed. The study variables included age, gender, tumor size, histological type, nuclear grade, level of IVC thrombus, estimated blood loss, transfusions, hospital stay and follow-up.

The entire cohort was divided into two groups based on the laterality of the renal tumor. The level of IVC involvement was stratified by the distal limit of the tumor thrombus: level-I involving the IVC at the level of the renal vein; level-II, the infra hepatic IVC; level-III, the retro-hepatic IVC; level-IV right atrium $(4,5)$. The surgical technique we use has been described in detail in a previous publication (6). We followed the patients post-surgery at one month and then every 3 months with a medical history, physical examination, metabolic panel and liver function test. A chest X-ray was obtained every 6 months and an abdominal CT scan every year.

Chi-square test and Fisher's exact test were used to compare categorical variables. The ANOVA test was used to compare continuous variables. For survival analysis, Kaplan-Meier method was used. The log-rank test was used to see for any difference in survival distributions. A two sided $\mathrm{p}$ value $\leq$ 0.05 was considered statistically significant. All the analyses were done using the SPSS software (Version 16).

\section{RESULTS}

In total 87 patients were included in the study. There were 47 men (54\%) and 40 women (46\%). Sixty patients $(69 \%)$ had RCC on the right side. The mean age (61 years) mean tumor size (10.25 $\pm 4 \mathrm{~cm}$ ), T stage, lymph node status and metastatic presentation were not significantly different between both groups (Table-1). A level-III or higher IVC thrombus was seen more often with a right sided RCC. Seventy-two of right side tumors compared to $41 \%$ of the left side tumors had a level-III or higher IVC thrombus $(p<0.001)$. Six $(10 \%)$ of right side tumors had level-I IVC thrombus compared to 13 $(48 \%)$ of left side tumors.

Table 1 - Clinical and pathological characteristics.

\begin{tabular}{lccc}
\hline & Right $(\mathrm{N}=60)$ & Left $(\mathrm{N}=27)$ & p Value \\
\hline Age in years (mean $\pm \mathrm{SD})$ & $62 \pm 12$ & $59 \pm 13$ & 0.38 \\
Men & 38 & 9 & 0.01 \\
Women & 22 & 18 & \\
Mean tumor size $(\mathrm{cm})( \pm \mathrm{SD})$ & $10.5 \pm 4$ & $10 \pm 5$ & 0.65 \\
Level $(\%)$ & & & \\
I & 6 & 3 & 0.0001 \\
II & 11 & 6 & \\
III & 34 & 5 & \\
IV & 9 & 4 & 0.55 \\
N+ & 14 & 2 & 0.7 \\
Metastases at presentation $(\mathrm{M} 1)$ & 7 & 3 & \\
\hline
\end{tabular}


The mean estimated blood loss and the mean number of blood transfusions was not significantly different between the groups (Table-2). The mean hospital stay was also similar. There were two perioperative deaths in the right side group, one due to cardiac arrhythmia and the other due to hepatic failure. There was one perioperative death in left side group due to pulmonary failure in a chronic obstructive pulmonary disease patient.

The mean follow-up was 22.5 months (3-99). There was no significant difference in the diseasefree and disease specific survival between the groups (Table-2) (Figure-1).

\section{COMMENT}

Although the human body displays distinct external symmetry, the internal anatomy is strikingly asymmetrical. The paired organs are typically asymmetrical and can differ in mass, position, blood supply, lymphatic drainage and relation to surrounding organs. This difference can play an important role in evolution of the disease process. The incidence of cancer itself may be marginally higher on one side. Breast cancer incidence is relatively higher on the left side (7). In genitourinary cancers, testicular neoplasms occur more commonly on the right side. The incidence of renal cancer does not significantly differ with the laterality of the tumor. The venous anatomy of the right kidney however is markedly different from the left kidney.

Most series on IVC thrombectomy have a higher number of patients with right side RCC. In our series, $69 \%$ patients with IVC thrombus had a right renal tumor. This may be due to an anatomically short right renal vein $(2-4 \mathrm{~cm})$ allowing a shorter path into the IVC and hence a higher incidence at the time of presentation. In a series of 93 patients with IVC thrombus, Staehler et al. reported that $81 \%$ had a right side renal tumor (8). In another series by Lambert et al. $64 \%$ had a right side tumor (9). In our series, a level-III or higher thrombus was seen more frequently with a right sided tumor. This can be explained by the fact that the left renal vein is typically $6-10 \mathrm{~cm}$. Assuming a similar rate of tumor thrombus growth for both the sides a right sided tumor thrombus grows into the intra-hepatic IVC earlier. Accurate identification of the anatomic level of IVC thrombus is important for planning the surgical approach (6).

At our institution, we have modified our surgical technique using principles derived from liver transplantation (6). We use a transabdominal approach with mobilization of the liver, which gives excellent exposure. When dealing with large left side renal tumors with IVC involvement we mobilize the stomach, spleen and pancreas en-bloc (10). Although

Table 2-Operative variables and follow-up.

\begin{tabular}{lccc}
\hline & $\begin{array}{c}\text { Right } \\
(\mathrm{N}=60)\end{array}$ & $\begin{array}{c}\text { Left } \\
(\mathrm{N}=27)\end{array}$ & p Value \\
\hline EBL (liters) & 1.6 & 2 & 0.58 \\
Transfusion (units) & $3.5 \pm 8$ & $4 \pm 6.5$ & 0.85 \\
Hospital stay (Days) ( $\pm \mathrm{SD})$ & $8.85 \pm 6.7$ & $8.9 \pm 5.7$ & 0.97 \\
Mean follow-up (months) & 24 & 19 & 0.27 \\
Recurrence & 16 & 6 & 0.80 \\
DS death & 26 & 7 & 0.20 \\
5 years disease free survival \pm SE (\%) & $63 \pm 7.5$ & $70 \pm 12$ & 0.94 \\
5 years disease specific survival \pm SE (\%) & $44 \pm 8$ & $52 \pm 14$ & 0.42 \\
\hline
\end{tabular}

$E B L=$ estimated blood loss; $D S=$ disease specific. 


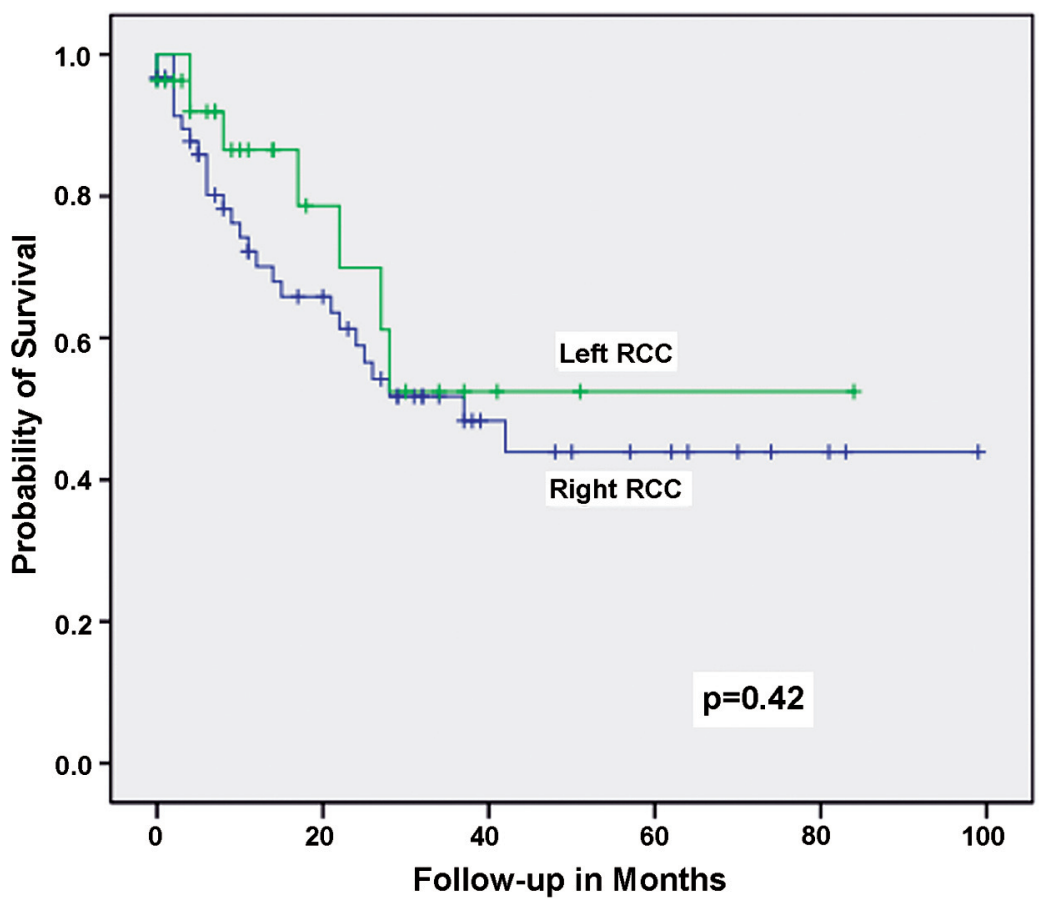

Figure 1 - Kaplan-Maier plot showing disease specific survival in right and left renal tumors undergoing radical nephrectomy and inferior vena cava thrombectomy.

the surgical procedure is to some extent different for IVC thrombectomy when the tumor is on right compared to left side, however in our study we did not find any significant difference in surgical outcome. The disease free and disease specific survival were also not significantly different between right and left side tumors. The prognostic impact of the level of thrombus remains controversial. Majority of current literature supports the view that the level of tumor thrombus does not influence survival $(11,12)$. This explains the similar long-term outcome for right and left sided tumors despite the right side tumors having a higher anatomic level of tumor thrombus.

Our study has some limitations. It is a retrospective study and it represents a single center experience.

\section{CONCLUSIONS}

Our results show that a level-III tumor thrombus is more frequently seen when the tumor is on right side compared to the left side. However, clinical and operative characteristics among the left and right sided tumors with IVC thrombus were not different. More significantly, recurrence rate and the survival did not differ with the laterality of the tumor.

\section{ACKNOWLEDGEMENTS}

Financial support from "CURED" and Mr. Vincent A. Rodriguez.

\section{CONFLICT OF INTEREST}

None declared.

\section{REFERENCES}

1. Jemal A, Siegel R, Ward E, Hao Y, Xu J, Murray T, et al.: Cancer statistics, 2008. CA Cancer J Clin. 2008; 58: 71-96. 
2. Wotkowicz C, Wszolek MF, Libertino JA: Resection of renal tumors invading the vena cava. Urol Clin North Am. 2008; 35: 657-71.

3. Marshall FF, Dietrick DD, Baumgartner WA, Reitz BA: Surgical management of renal cell carcinoma with intracaval neoplastic extension above the hepatic veins. J Urol. 1988; 139: 1166-72.

4. Neves RJ, Zincke H: Surgical treatment of renal cancer with vena cava extension. Br J Urol. 1987; 59: 390-5.

5. Ciancio G, Vaidya A, Savoie M, Soloway M: Management of renal cell carcinoma with level III thrombus in the inferior vena cava. J Urol. 2002; 168: 1374-7.

6. Ciancio G, Livingstone AS, Soloway M: Surgical management of renal cell carcinoma with tumor thrombus in the renal and inferior vena cava: the University of Miami experience in using liver transplantation techniques. Eur Urol. 2007; 51: 988-94; discussion 994-5.

7. Dane S, Yildirim S, Koc M, Aktan M, Gundogdu C: Asymmetries in breast cancer lateralization and both axillary lymph node number and metastatic involvement. Lymphology. 2008; 41: 75-9.
8. Staehler G, Brkovic D: The role of radical surgery for renal cell carcinoma with extension into the vena cava. J Urol. 2000; 163: 1671-5.

9. Lambert EH, Pierorazio PM, Shabsigh A, Olsson CA, Benson MC, McKiernan JM: Prognostic risk stratification and clinical outcomes in patients undergoing surgical treatment for renal cell carcinoma with vascular tumor thrombus. Urology. 2007; 69: 1054-8.

10. Ciancio G, Vaidya A, Shirodkar S, Manoharan M, Hakky T, Soloway M: En Bloc Mobilization of the Pancreas and Spleen to Facilitate Resection of Large Tumors, Primarily Renal and Adrenal, in the Left Upper Quadrant of the Abdomen: Techniques Derived from Multivisceral Transplantation. Eur Urol. 2009; 9. [Epub ahead of print]

11. Wagner B, Patard JJ, Méjean A, Bensalah K, Verhoest $\mathrm{G}$, Zigeuner R, et al.: Prognostic value of renal vein and inferior vena cava involvement in renal cell carcinoma. Eur Urol. 2009; 55: 452-9.

12. Kirkali Z, Van Poppel H: A critical analysis of surgery for kidney cancer with vena cava invasion. Eur Urol. 2007; 52: 658-62.

\section{Correspondence address:}

Dr. Mark S. Soloway

Professor and Chairman

Department of Urology

Univ. of Miami Miller Sch. of Medicine

P.O. Box 016960

Miami, FL 33101, USA

Fax: +1 305 243-4653

E-mail: msoloway@med.miami.edu

\section{EDITORIAL COMMENT}

The authors retrospectively analyzed patients who underwent radical nephrectomy and inferior vena cava (IVC) thrombectomy. In total 87 patients were included in the study. There were 47 men (54\%) and
40 women (46\%) and 60 (69\%) patients had RCC on the right side.

The results show that a level-III tumor thrombus is more frequently seen when the tumor is on right 
side compared to the left side and more significantly, recurrence rate and the survival did not differ with the laterality of the tumor. Clinical and operative characteristics among the left and right sided tumors with IVC thrombus were not different. More significantly, recurrence rate and the survival did not differ with the laterality of the tumor. These results are obvious since the venous anatomy of the right kidney is markedly different from the left kidney.

Dr. Antonio Augusto Ornellas Section of Urology National Institute of Cancer and Mario Kroeff Cancer Hospital

Rio de Janeiro, Brazil

E-mail:ornellasa@hotmail.com 